\title{
An Optimized Experimental Methodology for L-Band Signal Propagation Sentences Evaluation
}

\section{I. $A b b a$}

Department of Electrical / Electronic Technology School of Technology, Kano State Polytechnic, Kano Nigeria

ibrahimaba12950@yahoo.com

\author{
W. A.W. Z. Abidin, B. V. Pai
}

Department of Electrical and Electronic Engineering, Faculty of Engineering Universiti Malaysia Sarawak Sarawak, Malaysia

\begin{abstract}
Combining external patch antenna with Global Positioning System (GPS) portable device is necessary to control raw data quality degradation bonded with low signal strength when receiver is operating at low elevation angle. However, this work has designed an economic data collection system comprising developed adaptive graphical user interface and antenna gain enhancement module that manage the open sky GPS propagation data and characterize the signal-to-noise (SNR) as function of elevation and azimuth angles. The model which will used to characterize received SNR mobile satellite (MS) at Kano and Samarahan, show betterquality signal strength with the other one without external patch antenna, thus provide better services to MS users for this environment.
\end{abstract}

Keywords-SNR; GPS receiver; Mobile Satellite; Patch antenna;

\section{INTRODUCTION}

The signal performance of the mobile satellite (MS) is affected by many factors such as ionosphere effect, trees shadowed; building shadowed and multipath effect [1-5], but this experimental research will focus on the satellite signals for an open space environment in which the receiving satellite signal does not experience significant fading effect due to building or trees. Whereby an economical system for a data acquisition was designed and developed. From the detail reports on the previous studies conducted many are lacking the use of external single micro strip patch antenna (SMPA) in cooperates with handheld GPS receiver to acquired MS sentences particularly in Kota Samarahan and Kano.

The study frame work was summarized in Fig. 1 shows the experiment flow process and the research study was categorized into three stages, comprising; external antenna base, open space base and data acquisition laboratory base. The external antenna bases it the section whereby the selection, design and fabrication of $1.57542 \mathrm{GHz}$ antenna was conducted, follow by the open space section whereby the receiver and its cabling connection were exposed to open space environment and finally the acquisition lab where the GPS satellite sentences for propagation data were monitored and saves for later analysis [6], [7].

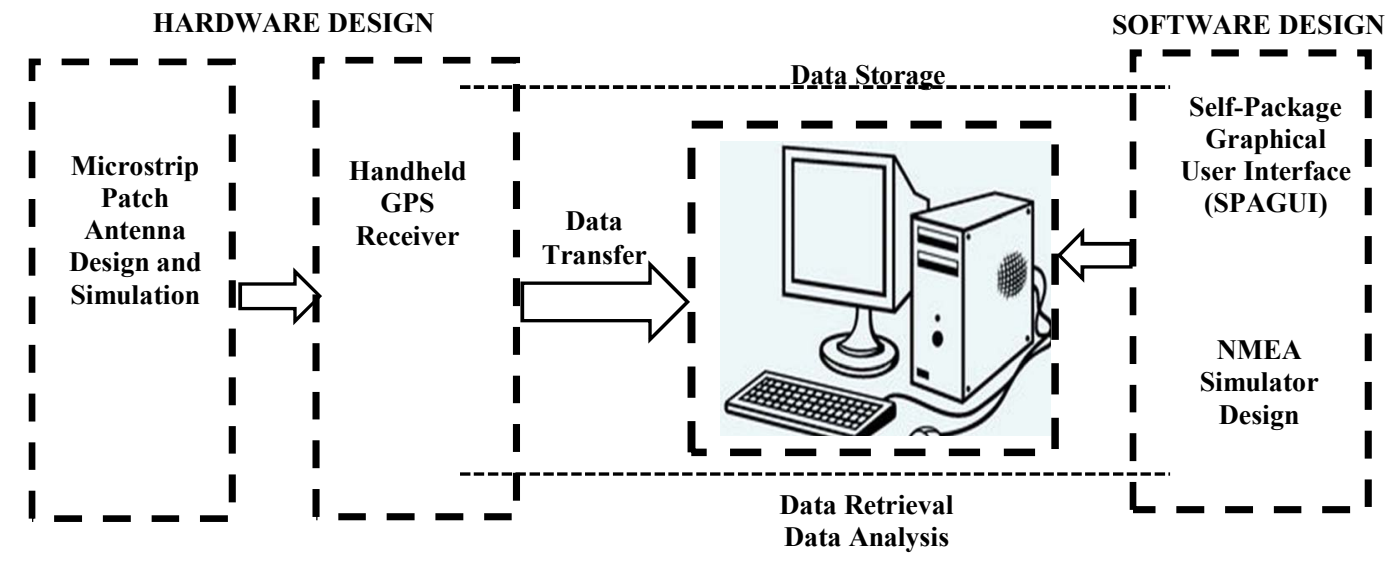

Fig. 1: Study flow frame work

A cost effective data acquisition system for L-band mobile satellite signal performance measurement was designed using

Global Positioning System (GPS) approach. These refer to the experimental setup of the equipment that is the connection done between the GPS receiver with a computer [8] and fabricated external patch antenna for GPS receiver. The satellite propagation data received from the GPS satellite will be recorded continuously with an updates rate of 2 seconds. The 